\title{
Flexural Analysis of Thick Isotropic Rectangular Plates Using Orthogonal Polynomial Displacement Functions
}

\author{
Onodagu P. Dinwoke, Aginam C. Henry, and Uzodinma C. Franklin
}

\begin{abstract}
This paper analysed the flexural behaviour of SSSS thick isotropic rectangular plates under transverse load using the Ritz method. It is assumed that the line that is normal to the mid-surface of the plate before bending does not remain the same after bending and consequently a shear deformation function $f(z)$ is introduced. A polynomial shear deformation function $f(z)$ was derived for this research. The total potential energy which was established by combining the strain energy and external work was subjected to direct variation to determine the governing equations for the in - plane and out-plane displacement coefficients. Numerical results for the present study were obtained for the thick isotropic SSSS rectangular plates and comparison of the results of this research and previous work done in literature showed good convergence. However, It was also observed that the result obtained in this present study are significantly upper bound as compared with the results of other researchers who employed the higher order shear deformation theory (HSDT), first order shear deformation theory (FSDT) and classical plate theory (CPT) theories for the in - plane and out of plane displacements at span - depth ratio of 4. Also, at a span - depth ratio of 100 and above, there was approximately no difference in the values obtained for the out of plane displacements and in-plane displacements between the CPT and the theory used in this study.
\end{abstract}

Key words - displacement, Ritz method, shear deformation, stress.

\section{INTRODUCTION}

A plate is said to be a flat structural component bound by two planes that are parallel to each other called faces and a cylindrical surface called an edge [1]. Plates are very important structural elements that have been in use for ages. In general, when plates are subjected to loading, they cause deflections on the plate. Plates have a similar load-bearing action with that of beams to a reasonable degree and therefore, depending on the flexural rigidity of the structures, plates can be approximated by a composition of beams.

Plates are of varying uses in the engineering industry. In recent times, plates and plated structures have been used in architectural structures, bridges, hydraulic structures, pavements, containers, airplanes, missiles, ships, and machine parts. The issues related to different combinations of edge conditions with regards to rectangular thick plates are infrequent in many engineering fields. Moreover, with the improvement as regards to modern industry, relatively more accurate and practical studies with regards to bending in

Submitted on December 03, 2021.

Published on December 29, 2021.

Onodagu P. Dinwoke, Nnamdi Azikiwe University, Awka, Anambra State, Nigeria.

(e-mail: pd.onodagu@unizik.edu.ng) plates are required.

The classical plate theory (CPT) is mostly used in analyzing thin plates. However, the classical plate theory assumes the transverse shear deformation to be zero and that the normal to the mid-plane remains straight before and after deformation. Making use of CPT to analyze thick plates may result in incorrect and/or wrong results. Consequent upon the deficiency of the classical plate theory, many researchers have developed series of theories to analyze thick plates by taking into account the shear deformation. Examples of such theories include Mindlin's first-order, Reddy's third-order, and Reissner's higher-order theories. However, these theories have two more unknown variables in comparison with the classical plate theory [2], [3].

Flexural analysis of thick plates from some previous works in literature was based on trigonometric displacement functions, u, v, and w. Upon analysis and design of thick plates using these trigonometric displacement functions, the use of the Fourier series becomes unavoidable resulting in rigorous and complicated mathematical calculations which lead to idealizing most of these thick plates as thin plates thus, leading to erroneous designs which are not dependable and could lead to failure. [4]. Consequently, this research seeks to present a direct, simplified, and precise approach through the use of the Ritz energy method resulting in Polynomial Displacement Functions to solve the problems of thick rectangular isotropic plates under transverse loading.

\section{THEORETICAL FRAMEWORK}

Soldatos [5] presented a general two-dimensional theory suitable for the static and/or dynamic analysis of a transverse shear deformable plate consisting of a homogeneous, monoclinic, linearly elastic material and subjected to any type of shear tractions at its lateral planes. He proposed a function given as:

$$
f(z)=\left[z \cosh \left(\frac{1}{2}\right)-t \sinh \left(\frac{z}{t}\right)\right]
$$

In non-dimensional form; $z=s t$

Where ' $\mathrm{s}$ ' is a non-dimensional parameter along $\mathrm{z}$-axis, and ' $t$ ' is the thickness of the plate.

From the [5] model graph $(-0.5 \leq s \leq 0.5)$ with an interval of 0.025 represented graphically and the equation of the curve can be expressed in polynomial form as:

Aginam C. Henry, Nnamdi Azikiwe University, Awka, Anambra State, Nigeria.

(e-mail: ch.aginam@ ${ }^{@}$ unizik.edu.ng)

Uzodinma C. Franklin, Nnamdi Azikiwe University, Awka, Anambra State, Nigeria.

(e-mail: fc.uzodinma@ ${ }^{@}$ unizik.edu.ng). 
$f(z)=t\left[\left(\frac{z}{t}\right)-\frac{13}{10}\left(\frac{z}{t}\right)^{3}\right]$

This can be simplified as:

$$
f(z)=z\left[1-\frac{13}{10}\left(\frac{z}{t}\right)^{2}\right]
$$

\section{A. Assumptions}

In this study, it is assumed the out-of-plane displacement (deflection), $\mathrm{w}$ is only differentiable in $\mathrm{x}$ and $\mathrm{y}$ axes. Consequently, the vertical strain, $\varepsilon_{z}=0$ and the effect of the out-of-plane normal stress on the response of the plate is small with respect to the other stresses. Thus, it is neglected. That is $\sigma_{z}=0$. However, in contrast to the CPT, it is also assumed that the vertical line that is initially normal to the middle surface of the plate before bending becomes parabolic after bending.

\section{B. Kinematic Relations}

In this shear deformation theory, in-plane displacements, $u$ and $\mathrm{v}$ are defined mathematically as:

$$
u=u_{c}+u_{s} ; v=v_{c}+v_{s}
$$

$u_{s}$ and $v_{s}$ are the shear deformation components of the inplane displacements and are defined mathematically as:

$$
u_{s}=f(z) . \emptyset_{x} ; v_{s}=f(z) \cdot \emptyset_{y}
$$

where; $\varnothing x=$ shear rotation in $x-$ direction and $\emptyset y=$ shear rotation in $y$-direction.

According to the classical plate theory (CPT), the in -plane displacements $u_{c}$ and $v_{c}$ can be expressed as:

$$
u_{c}=-z \theta_{c x}=-z \frac{d w}{d x} ; v_{c}=-z \theta_{c y}=-z \frac{d w}{d y}
$$

Therefore,

$$
u=-z \frac{\partial w}{\partial x}+f(z) \cdot \emptyset_{x} ; v=-z \frac{\partial w}{\partial y}+f(z) \cdot \emptyset_{y}
$$

\section{Strain-Displacement Relations}

Strain - displacement relations suitable for an isotropic and homogenous material in three dimensions were developed by Ventsel and Krauthammer [6] and applying the assumption that the vertical strain, $\varepsilon_{z}=0$ to the strain - displacement we have:

$$
\begin{gathered}
\varepsilon_{x}=\frac{\mathrm{d} u}{d x}=-z \frac{\partial^{2} w}{\partial x^{2}}+f(z) \cdot \frac{\partial \emptyset_{x}}{\partial x} ; \varepsilon_{y}=\frac{d v}{d y}=-z \frac{\partial^{2} w}{\partial y^{2}}+ \\
f(z) \cdot \frac{\partial \emptyset_{y}}{\partial y} \quad \text { (7) } \quad \gamma_{x y}=\frac{d u}{d y}+\frac{d v}{d x}=-z \frac{\partial^{2} w}{\partial x \partial y}+f(z) \cdot \frac{\partial \emptyset_{x}}{\partial y}- \\
z \frac{\partial^{2} w}{\partial x \partial y}+f(z) \cdot \frac{\partial \emptyset_{y}}{\partial x}=-2 z \frac{\partial^{2} w}{\partial x \partial y}+f(z) \cdot \frac{\partial \emptyset_{x}}{\partial y}+f(z) \\
\gamma_{x z}=\frac{d u}{d z}+\frac{d w}{d x}=-\frac{\partial w}{\partial x}+f(z) \cdot \frac{\partial \emptyset_{x}}{\partial z}+\frac{d w}{d x}=f(z) \cdot \frac{\partial \emptyset_{x}}{\partial z} \\
\gamma_{y z}=\frac{d v}{d z}+\frac{d w}{d y}=-\frac{\partial w}{\partial y}+f(z) \cdot \frac{\partial \emptyset_{y}}{\partial z}+\frac{d w}{d y}=f(z) \cdot \frac{\partial \emptyset_{y}}{\partial z}
\end{gathered}
$$

where $u, v, w$ are displacements in $\mathrm{x}, \mathrm{y}$, and $\mathrm{z}$ directions respectively.

\section{Constitutive (Stress Strain) Relations}

Ugural [7] expressed a relationship for stress and strain by the generalized Hooke's law for a three-dimensional state of stress valid for an isotropic homogenous material, upon simplification and necessary substitution into the constitutive equations, we obtain the stress - displacement relations as follows:

$\sigma_{x}=\frac{E}{1-\mu^{2}}\left[-Z \frac{\partial^{2} w}{\partial x^{2}}+f(z) \cdot \frac{\partial \emptyset_{x}}{\partial x}+\mu\left(-z \frac{\partial^{2} w}{\partial y^{2}}+f(z) \cdot \frac{\partial \emptyset_{y}}{\partial y}\right)\right]$

$\sigma_{y}=\frac{E}{1-\mu^{2}}\left[\mu\left(-z \frac{\partial^{2} w}{\partial x^{2}}+f(z) \cdot \frac{\partial \emptyset_{x}}{\partial x}\right)+\left(-z \frac{\partial^{2} w}{\partial y^{2}}+f(z)\right.\right.$.

$\left.\left.\frac{\partial \emptyset_{y}}{\partial y}\right)\right]$

$\tau_{x y}=\frac{E(1-\mu)}{2\left(1-\mu^{2}\right)}\left[-2 z \frac{\partial^{2} w}{\partial x \partial y}+f(z) \cdot \frac{\partial \emptyset_{x}}{\partial y}+f(z) \cdot \frac{\partial \emptyset_{y}}{\partial x}\right]$

$\tau_{x z}=\frac{E(1-\mu)}{2\left(1-\mu^{2}\right)}\left[f(z) \cdot \frac{\partial \emptyset_{x}}{\partial z}\right] ; \tau_{y z}=\frac{E(1-\mu)}{2\left(1-\mu^{2}\right)}\left[f(z) \cdot \frac{\partial \emptyset_{y}}{\partial z}\right]$

where $\mu$ is the Poisson ratio.

\section{E. Strain Energy $(U)$}

The Strain energy U, stored in a continuum of the plate was given by [7] as the product of stress, strain and volume of the continuum. $\mathrm{U}$ is given mathematically as shown:

$$
U=\frac{1}{2} \int_{x} \int_{y}\left[\int_{z} \sigma \cdot \varepsilon d z\right] d x d y
$$

Considering strain and stress in $\mathrm{x}, \mathrm{y}, \mathrm{z}$ direction of an elastic body, the strain energy is expressed as shown:

$$
\begin{aligned}
& U=\frac{1}{2} \int_{x} \int_{y}\left[\int _ { - t / 2 } ^ { t / 2 } \left(\sigma_{x} \varepsilon_{x}+\sigma_{y} \varepsilon_{y}+\tau_{x y} \gamma_{x y}+\tau_{x z} \gamma_{x z}+\right.\right. \\
& \left.\left.\tau_{y z} \gamma_{y z}\right) d z\right] d x d y
\end{aligned}
$$

Let the sum of the components of the strain energy of the thick plate be $U_{1}$. Therefore $U_{1}$ can be expressed as:

$$
U_{1}=\sigma_{x} \varepsilon_{x}+\sigma_{y} \varepsilon_{y}+\tau_{x y} \gamma_{x y}+\tau_{x z} \gamma_{x z}+\tau_{y z} \gamma_{y z}
$$

Substituting the stress and strain components and let the domain of $\mathrm{x}$ and $\mathrm{y}$ be $=a R ; y=b Q$; also, let $\propto=\frac{a}{t}$ and $p=$ $\frac{b}{a}$, upon simplification, we obtain:

$$
\begin{gathered}
U=\frac{1}{2} \int_{x} \int_{y}\left[\int _ { - \frac { t } { 2 } } ^ { \frac { t } { 2 } } \left(\sigma_{x} \varepsilon_{x}+\sigma_{y} \varepsilon_{y}+\tau_{x y} \gamma_{x y}+\tau_{x z} \gamma_{x z}+\right.\right. \\
\left.\left.\tau_{y z} \gamma_{y z}\right) d z\right] d x d y=\frac{D}{2} \int_{x} \int_{y}\left[\left[K_{1}\left(\frac{\partial^{2} w}{\partial x^{2}}\right)^{2}-2 K_{2} \cdot \frac{\partial \emptyset_{x}}{\partial x}\left(\frac{\partial^{2} w}{\partial x^{2}}\right)+\right.\right. \\
\left.K_{3} \cdot\left(\frac{\partial \emptyset_{x}}{\partial x}\right)^{2}\right]+\left[2 K_{1}\left(\frac{\partial^{2} w}{\partial x \partial y}\right)^{2}-2 K_{2} \cdot \frac{\partial \emptyset_{x}}{y} \frac{\partial^{2} w}{\partial x \partial y}-2 K_{2} \cdot\right. \\
\left.\frac{\partial \emptyset_{y}}{\partial x} \frac{\partial^{2} w}{\partial x \partial y}\right]+\left[K_{1}\left(\frac{\partial^{2} w}{\partial y^{2}}\right)^{2}-2 K_{2} \frac{\partial \emptyset_{y}}{\partial y} \frac{\partial^{2} w}{\partial y^{2}}+K_{3}\left(\frac{\partial \emptyset_{y}}{\partial y}\right)^{2}\right]+(1+ \\
\mu)\left[K_{3} \cdot \frac{\partial \emptyset_{y}}{\partial x} \frac{\partial \emptyset_{x}}{\partial y}\right]+\frac{(1-\mu)}{2}\left[K_{3}\left(\frac{\partial \emptyset_{x}}{\partial y}\right)^{2}+K_{3}\left(\frac{\partial \emptyset_{y}}{\partial x}\right)^{2}\right]+\frac{(1-\mu)}{2} .
\end{gathered}
$$


$\frac{\alpha^{2}}{a^{2}} K_{4} \cdot\left[\emptyset_{x}^{2}+\emptyset_{y}^{2}\right] d x d y$

where $D=\frac{E t^{3}}{12\left(1-\mu^{2}\right)}$.

Using $f(z)=z\left[1-\frac{13}{10}\left(\frac{z}{t}\right)^{2}\right], \mathrm{K}_{\mathrm{i}}$ values upon calculation can be summarized below:

$$
K_{1}=1, K_{2}=0.805, K_{3}=0.655 \text { and } K_{4}=6.48
$$

\section{F. External Work, $V$ on the Thick Plate}

The external work due to lateral load uniformly distributed on the plate, $\mathrm{q}$ is given as:

$$
\mathrm{V}=-\mathrm{q} \iint_{\mathrm{xy}} \mathrm{wdxdy}
$$

where $\mathrm{q}=$ lateral load and $\mathrm{w}=$ deflection.

\section{G. Total Potential Energy}

Total potential energy ' $\Pi$ ', of the thick plate is the sum of strain energy, $\mathrm{U}$ and external work, $\mathrm{V}$.

$$
\Pi=U+V=\frac{D}{2} \int_{x} \int_{y}\left[\left[K_{1}\left(\frac{\partial^{2} w}{\partial x^{2}}\right)^{2}-2 K_{2} \cdot \frac{\partial \emptyset_{x}}{\partial x}\left(\frac{\partial^{2} w}{\partial x^{2}}\right)+\right.\right.
$$

$\left.K_{3} \cdot\left(\frac{\partial \emptyset_{x}}{\partial x}\right)^{2}\right]+\left[2 K_{1}\left(\frac{\partial^{2} w}{\partial x \partial y}\right)^{2}-2 K_{2} \cdot \frac{\partial \emptyset_{x}}{\partial y} \frac{\partial^{2} w}{\partial x \partial y}-2 K_{2}\right.$.

$\left.\frac{\partial \emptyset_{y}}{\partial x} \frac{\partial^{2} w}{\partial x \partial y}\right]+\left[K_{1}\left(\frac{\partial^{2} w}{\partial y^{2}}\right)^{2}-2 K_{2} \frac{\partial \emptyset_{y}}{\partial y} \frac{\partial^{2} w}{\partial y^{2}}+K_{3}\left(\frac{\partial \emptyset_{y}}{\partial y}\right)^{2}\right]+(1+$

н) $\left[K_{3} \cdot \frac{\partial \emptyset_{y}}{\partial x} \frac{\partial \emptyset_{x}}{\partial y}\right]+\frac{(1-\mu)}{2}\left[K_{3}\left(\frac{\partial \emptyset_{x}}{\partial y}\right)^{2}+K_{3}\left(\frac{\partial \emptyset_{y}}{\partial x}\right)^{2}\right]+\frac{(1-\mu)}{2}$.

$\left.\frac{\alpha^{2}}{a^{2}} K_{4}\left[\varnothing_{x}^{2}+\emptyset_{y}^{2}\right]\right] d x d y-q \int_{x} \int_{y} w d x d y$

\section{H. Formulation of the Direct Governing Simultaneous Equations}

Let

$$
w=w_{x} \cdot w_{y} ; \emptyset_{x}=\emptyset_{x y} \cdot \emptyset_{x x} ; \emptyset_{y}=\emptyset_{y x} \cdot \emptyset_{y y}
$$

Let

$$
n_{1}+n_{2}=1 n_{3}+n_{4}=1 n_{5}+n_{6}=1
$$

Differentiating the total energy equation with respect to $\mathrm{w}$ and collecting like terms, we obtain:

$$
\begin{aligned}
& \frac{d \Pi}{d w}=\int_{0}^{1} \int_{0}^{1}\left[\left(\frac{\partial^{4} w}{\partial R^{4}} n_{1}+\frac{a^{4} q}{D}\right)+\left(\frac{\partial^{4} w}{P^{2} \partial R^{2} \partial Q^{2}}-\mathrm{ak}_{2}\right.\right. \\
& \left.\frac{\partial^{3} \emptyset_{x}}{P^{2} \partial R \partial Q^{2}}\right)+\left(\frac{\partial^{4} w}{P^{2} \partial R^{2} \partial Q^{2}} n_{3}-a k_{2} \cdot \frac{\partial^{3} \emptyset_{y}}{P \partial R^{2} \partial Q}\right)+\left(\frac{\partial^{4} w}{P^{4} \partial Q^{4}} n_{5}+\right. \\
& \left.\frac{\partial^{4} w}{P^{2} \partial R^{2} \partial Q^{2}} n_{4}\right)+\left(\frac{\partial^{4} w}{\partial R^{4}} n_{2}-a k_{2} \cdot \frac{\partial^{3} \emptyset_{x}}{\partial R^{3}}\right)+\left(\frac{\partial^{4} w}{P^{4} \partial Q^{4}} n_{6}-\right. \\
& \left.\left.a k_{2} \frac{\partial^{3} \emptyset_{y}}{P^{3} \partial Q^{3}}\right)\right] \mathrm{dRdQ}=0
\end{aligned}
$$

For the equation to be true, each bracket must identically be equal to zero. Executing closed domain integration for the components in the bracket, letting $w=J_{1} h$ we obtain:

$$
\begin{aligned}
\emptyset_{x} & =\emptyset_{x y} \cdot \emptyset_{x x}=\frac{1}{\mathrm{a}^{2} k_{2}^{2}} \frac{\int_{0}^{1}\left(\frac{\partial^{2} w_{x}}{\partial R^{2}}\right) d R}{\int_{0}^{1}\left(\frac{\partial \phi_{x x}}{\partial R}\right) d Q} \cdot \frac{\int_{0}^{1}\left(\frac{\partial^{2} w_{y}}{\partial Q^{2}}\right) d Q}{\int_{0}^{1}\left(\frac{\partial^{2} \emptyset_{x y}}{\partial Q^{2}}\right) d Q} \frac{\partial w}{\partial R}=C_{a} . \\
J_{1} \frac{\partial h}{\partial x} & =J_{2} \frac{\partial h}{\partial x}
\end{aligned}
$$

$$
\emptyset_{y}=\emptyset_{y x} . \emptyset_{y y}=\frac{n_{3}^{2}}{P^{2} \mathrm{a}^{2} k_{2}^{2}} \frac{\int_{0}^{1}\left(\frac{\partial^{2} w_{y}}{\partial Q^{2}}\right) d R}{\int_{0}^{1}\left(\frac{\partial \phi_{y y}}{\partial Q}\right) d Q} \cdot \frac{\int_{0}^{1}\left(\frac{\partial^{2} w_{x}}{\partial R^{2}}\right) d R}{\int_{0}^{1}\left(\frac{\partial^{2} \emptyset_{y x}}{\partial R^{2}}\right) d R} \frac{\partial w}{\partial Q}=
$$

$C_{\mathrm{b}} \cdot J_{1} \frac{\partial h}{\partial y}=J_{3} \frac{\partial h}{\partial y}$

where $J_{2}=C_{a} \cdot J_{1}$ and $J_{3}=C_{\mathrm{b}} \cdot J_{1} \cdot J_{1}, J_{2}$, and $J_{3}$ are constants to be determined.

Also, the approximate polynomial for the deflection "w" of an isotropic rectangular plate for flexural analysis can be obtained as:

$$
\begin{aligned}
& w=w_{x} \cdot w_{y}=\left(a_{0}+a_{1} R+a_{2} R^{2}+a_{3} R^{3}+a_{4} R^{4}\right) \\
& \left(b_{0}+b_{1} Q+b_{2} Q^{2}+b_{3} Q^{3}+b_{4} Q^{4}\right)
\end{aligned}
$$

Substituting $\emptyset_{x}$ and $\emptyset_{y}$ into $\Pi$ and further simplification we obtain:

$$
\begin{aligned}
& \Pi=\frac{D a b}{2 a^{4}} \int_{0}^{1} \int_{0}^{1}\left[\left(J_{1}^{2} k_{1}-2 J_{1} J_{2} k_{2}+J_{2}^{2} k_{3}\right)\left(\frac{d^{2} h}{d R^{2}}\right)^{2}+\right. \\
& \left(J_{1}^{2} k_{1}-2 J_{1} J_{3} k_{2}+J_{3}^{2} k_{3}\right) \frac{1}{P^{4}}\left(\frac{d^{2} h}{d Q^{2}}\right)^{2}+\left(2 J_{1}^{2} k_{1}-2 J_{1} J_{2} k_{2}-\right. \\
& \left.2 J_{1} J_{3} k_{2}\right) \cdot \frac{1}{P^{2}}\left(\frac{d^{2} h}{d R^{2}} \cdot \frac{d^{2} h}{d Q^{2}}\right)+(1+\mu) J_{2} J_{3} k_{3} \cdot \frac{1}{P^{2}}\left(\frac{d^{2} h}{d R^{2}} \cdot \frac{d^{2} h}{d Q^{2}}\right)+ \\
& {\left[\left(\frac{1-\mu}{2}\right)\left(J_{2}^{2} k_{3}+J_{3}^{2} k_{3}\right)\right] \cdot \frac{1}{P^{2}}\left(\frac{d^{2} h}{d R^{2}} \cdot \frac{d^{2} h}{d Q^{2}}\right)+\propto^{2}\left(\frac{1-\mu}{2}\right) \cdot} \\
& \left.\left(J_{2}^{2} k_{4}\right)\left(\frac{d h}{d R}\right)^{2}+\frac{\alpha^{2}}{P^{2}}\left(\frac{1-\mu}{2}\right)\left(J_{3}^{2} k_{4}\right)\left(\frac{d h}{d Q}\right)^{2}\right] d R d Q- \\
& q a b J_{1} \int_{0}^{1} \int_{0}^{1}[\mathrm{~h}] \partial R \partial Q
\end{aligned}
$$

Applying Ritz method of total energy minimization:

$$
\frac{d \Pi}{d J_{1}}=0 ; \frac{d \Pi}{d J_{2}}=0 ; \frac{d \Pi}{d J_{3}}=0
$$

where $J_{1}, J_{2}$ and $J_{3}$ are coefficients of displacements for deflection, rotation in $\mathrm{x}$ axis and rotation in $\mathrm{y}$ axis respectively.

Expansion and simplification of these equations yield:

$$
\begin{aligned}
& \frac{D}{a^{4}} \int_{0}^{1} \int_{0}^{1}\left[\left(J_{1} k_{1}-J_{2} k_{2}\right)\left(\frac{d^{2} h}{d R^{2}}\right)^{2}+\left(J_{1} k_{1}-\right.\right. \\
& \left.J_{3} k_{2}\right) \frac{1}{P^{4}}\left(\frac{d^{2} h}{d Q^{2}}\right)^{2}+\left(2 J_{1} k_{1}-J_{2} k_{2}-J_{3} k_{2}\right) \cdot \frac{1}{P^{2}}\left(\frac{d^{2} h}{d R^{2}}\right. \\
& \left.\left.\frac{d^{2} h}{d Q^{2}}\right)\right] d R d Q=q \int_{0}^{1} \int_{0}^{1}[h] \partial R \partial Q
\end{aligned}
$$

$$
\begin{gathered}
\frac{D}{a^{4}} \int_{0}^{1} \int_{0}^{1}\left[\left(-J_{1} k_{2}+J_{2} k_{3}\right)\left(\frac{d^{2} h}{d R^{2}}\right)^{2}+\left(-J_{1} k_{2}\right) \cdot \frac{1}{P^{2}}\left(\frac{d^{2} h}{d R^{2}} .\right.\right. \\
\left.\frac{d^{2} h}{d Q^{2}}\right)+\frac{(1+\mu)}{2} \cdot J_{3} k_{3} \frac{1}{P^{2}}\left(\frac{d^{2} h}{d R^{2}} \cdot \frac{d^{2} h}{d Q^{2}}\right)+\left(\frac{1-\mu}{2}\right)\left(J_{2} k_{3}\right) \cdot \frac{1}{P^{2}}\left(\frac{d^{2} h}{d R^{2}} .\right. \\
\left.\left.\frac{d^{2} h}{d Q^{2}}\right)+\propto^{2}\left(\frac{1-\mu}{2}\right) \cdot\left(J_{2} k_{4}\right)\left(\frac{d h}{d R}\right)^{2}\right] d R d Q=0 \\
\frac{D}{a^{4}} \int_{0}^{1} \int_{0}^{1}\left[\left(-J_{1} k_{2}+J_{3} k_{3}\right) \frac{1}{P^{4}}\left(\frac{d^{2} h}{d Q^{2}}\right)^{2}+\left(-J_{1} k_{2}\right) \cdot \frac{1}{P^{2}}\left(\frac{d^{2} h}{d R^{2}} .\right.\right. \\
\left.\frac{d^{2} h}{d Q^{2}}\right)+\frac{(1+\mu)}{2} J_{2} k_{3} \cdot \frac{1}{P^{2}}\left(\frac{d^{2} h}{d R^{2}} \cdot \frac{d^{2} h}{d Q^{2}}\right)+\left[\left(\frac{1-\mu}{2}\right)\left(J_{3} k_{3}\right)\right] \cdot \frac{1}{P^{2}}\left(\frac{d^{2} h}{d R^{2}} .\right.
\end{gathered}
$$


$\left.\left.\frac{d^{2} h}{d Q^{2}}\right)+\frac{\alpha^{2}}{P^{2}}\left(\frac{1-\mu}{2}\right)\left(J_{3} k_{4}\right)\left(\frac{d h}{d Q}\right)^{2}\right] d R d Q=0$

Let,

$$
\begin{aligned}
& m_{1}=\int_{0}^{1} \int_{0}^{1}\left(\frac{\partial^{2} h}{\partial R^{2}}\right)^{2} \partial R \partial Q ; m_{2} \\
& =\int_{0}^{1} \int_{0}^{1}\left(\frac{\partial^{2} h}{\partial R^{2}} \cdot \frac{\partial^{2} h}{\partial Q^{2}}\right) \partial R \partial Q ; m_{3} \\
& =\int_{0}^{1} \int_{0}^{1}\left(\frac{\partial^{2} h}{\partial Q^{2}}\right)^{2} \partial R \partial Q \\
& m_{4}=\int_{0}^{1} \int^{1}\left(\frac{\partial \mathrm{h}}{\partial \mathrm{R}}\right)^{2} \partial R \partial Q ; \\
& m_{5}=\int_{0}^{1} \int_{0}^{1}\left(\frac{\partial \mathrm{h}}{\partial \mathrm{Q}}\right)^{2} \partial R \partial Q ; \\
& m_{6}=\int_{0}^{1} \int_{0}^{1} h \partial R \partial Q
\end{aligned}
$$

Substituting the values of $m_{1}, m_{2}, m_{3}, m_{4}$ and $m_{5}$, from (32) into (29), (30) and (31) and thus expressing same in matrix form:

$$
\left[\begin{array}{lll}
A_{11} & A_{12} & A_{13} \\
A_{21} & A_{22} & A_{23} \\
A_{31} & A_{32} & A_{33}
\end{array}\right] \cdot\left[\begin{array}{l}
J_{1} \\
J_{2} \\
J_{3}
\end{array}\right]=\frac{a^{4}}{D}\left[\begin{array}{c}
\mathrm{q} m_{6} \\
0 \\
0
\end{array}\right]
$$

Where

$$
\begin{aligned}
& A_{11}=k_{1}\left(m_{1}+\frac{2 m_{2}}{P^{2}}+\frac{m_{3}}{P^{4}}\right) ; \\
& A_{12}=A_{21}=-k_{2}\left(m_{1}+\frac{m_{2}}{P^{2}}\right) \\
& A_{13}=A_{31}=-k_{2}\left(\frac{m_{2}}{P^{2}}+\frac{m_{3}}{P^{4}}\right) ; \\
& A_{23}=A_{32}=\left(\frac{1+\mu}{2 P^{2}}\right) m_{2} k_{3} \\
& A_{22}=m_{1} k_{3}+\left(\frac{1-\mu}{2 P^{2}}\right) m_{2} k_{3}+\left(\frac{1-\mu}{2}\right) \propto^{2} m_{4} k_{4} \\
& A_{33}=\left(\frac{1-\mu}{2 P^{2}}\right) m_{2} k_{3}+\frac{m_{3}}{P^{4}} k_{3}+\left(\frac{1-\mu}{2 P^{2}}\right) \propto^{2} m_{5} k_{4} ;
\end{aligned}
$$

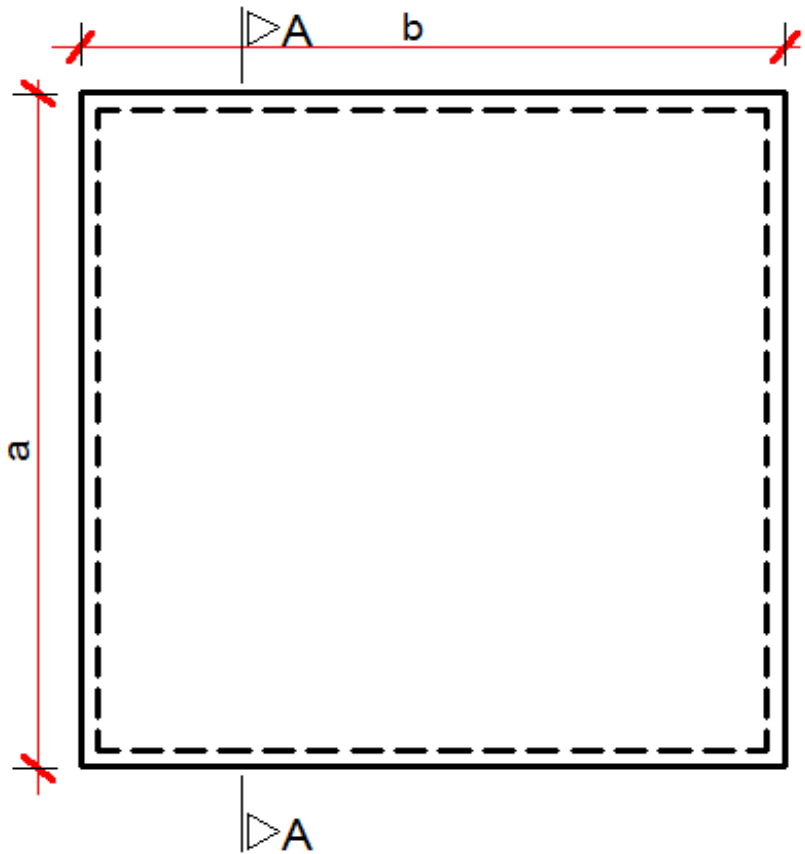

$$
m_{6}=\int_{0}^{1} \int_{0}^{1} h \partial R \partial Q
$$

\section{RESULTS}

Simply supported (SS) edge condition: The approximate polynomial for the deflection " $w$ " of an isotropic rectangular load for flexural analysis is presented as:

$$
\begin{aligned}
& w=w_{x} \cdot w_{y}=\left(a_{0}+a_{1} R+a_{2} R^{2}+a_{3} R^{3}+a_{4} R^{4}+\right. \\
& \cdots) \cdot\left(b_{0}+b_{1} Q+b_{2} Q^{2}+b_{3} Q^{3}+b_{4} Q^{4}+\cdots\right)
\end{aligned}
$$

The boundary conditions for SS edge are as follows:

a. At $\mathrm{R}=0$ and at $\mathrm{R}=1$, the deflection $w_{x}=0$.

b. At $R=0$ and at $\mathrm{R}=1$, Bending Moment $=0$, i.e. $\frac{\partial^{2} w_{x}}{\partial \mathrm{R}^{2}}=$

Substituting conditions

$$
\begin{aligned}
& a_{0}=0 ; a_{2}=0 ; a_{3}=-2 a_{4} \text { and } a_{1}=a_{4} \\
& w_{x}=a_{4}\left(R-2 R^{3}+R^{4}\right)
\end{aligned}
$$

In the same manner, repeating the same procedure for simply supported edge condition in the $\mathrm{x}$-direction for the $\mathrm{y}$ direction, we obtain a similar answer as expressed in (40b):

$$
w_{y}=b_{4}\left(Q-2 Q^{3}+Q^{4}\right)
$$

\section{A. SSSS Rectangular Plate Particular Shape Function}

Hence the deflection equation is obtained by multiplying (38) and (39).

$$
w=a_{4}\left(R-2 R^{3}+R^{4}\right) \cdot b_{4}\left(Q-2 Q^{3}+Q^{4}\right)
$$

However, $\quad$ since $\quad \mathrm{w}=A h=A\left(R-2 R^{3}+R^{4}\right) \cdot(Q-$ $\left.2 Q^{3}+Q^{4}\right)$

Where $A=a_{4} b_{4}$ And $h=\left(R-2 R^{3}+R^{4}\right) \cdot\left(Q-2 Q^{3}+\right.$ $\left.Q^{4}\right)$

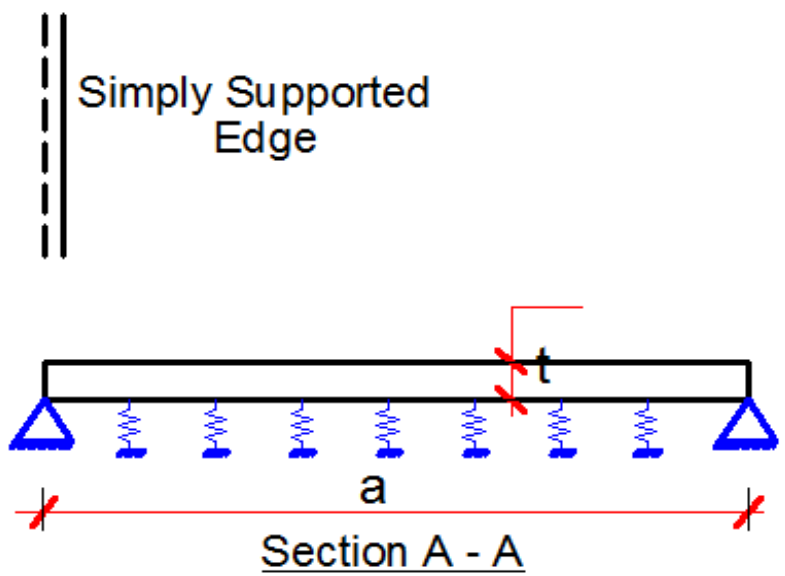

Fig. 1. SSSS rectangular plate. 
$h$ is the shape function for SSSS thick plate, 'A' is the coefficient of deflection. Upon substitution and simplification, we obtain:

$m_{1}=0.23619 ; \quad m_{2}=0.23592 ; m_{3}=0.23619 ; \quad m_{4}=0.0239 ;$ $m_{5}=0.0239 ; m_{6}=0.04$

And as already established $K_{1}=1, K_{2}=0.805 K_{3}=$ $0.655 K_{4}=6.48$ and $\propto=\frac{a}{t}$ and $p=\frac{b}{a}$.

Upon simplification of (33) the constants $j_{1}, j_{2}$ and $j_{3}$ can be obtained for different span to thickness ratio $\propto$, and thus, the deflection and displacements can also be determined.

However, in order to be able to compare results obtained in this present research with that of other researchers, the components of displacement and stresses will be presented herein as stated by [8] as:

$$
\begin{gathered}
\bar{w}=\frac{100 \mathrm{E} t^{3}}{\mathrm{q} a^{4}} w \\
\overline{(u,} \bar{v})=\frac{\mathrm{E} t^{2}}{\mathrm{q} a^{3}}(u, v)\left(\bar{\sigma}_{x}, \bar{\sigma}_{y}\right)=\frac{t^{2}}{\mathrm{q} a^{2}}\left(\sigma_{x}, \sigma_{y}\right)
\end{gathered}
$$

$$
\bar{\tau}_{x y}=\frac{t^{2}}{\mathrm{q} a^{2}} \tau_{x y} \quad\left(\bar{\tau}_{z x}, \bar{\tau}_{y z}\right)=\frac{t}{\mathrm{q} a}\left(\tau_{z x}, \tau_{y z}\right)
$$

\section{B. Numerical Example}

The deflection at the center of SSSS isotropic thick plate, the in-plane stresses, and the vertical shear stresses at the edges of the plate for different span to depth ratio corresponding to different values of $\frac{b}{a}$ subjected to a uniformly distributed load, $\mathrm{q}$ and $\mu=0.3$ is shown in Tables I, II and III.

Results obtained for the non-dimensional form of deflection $(\bar{w})$, displacement, $(\overline{\boldsymbol{u}})$ and stress $\left(\overline{\boldsymbol{\tau}}_{\boldsymbol{z} \boldsymbol{x}}\right)$ for each span to thickness ratio $\left(\propto=\frac{a}{t}\right)$ of SSSS with respect to the different aspect ratios ( $p=\frac{b}{a}$ ) as stipulated in Tables I, II and III can be expressed graphically as in Fig. 2, 3 and 4 respectively.

TABLE I: NUMERICAL RESULTS FOR THE NON-DIMENSIONAL FORM OF DISPLACEMENTS AND STRESSES FOR SSSS PLATES

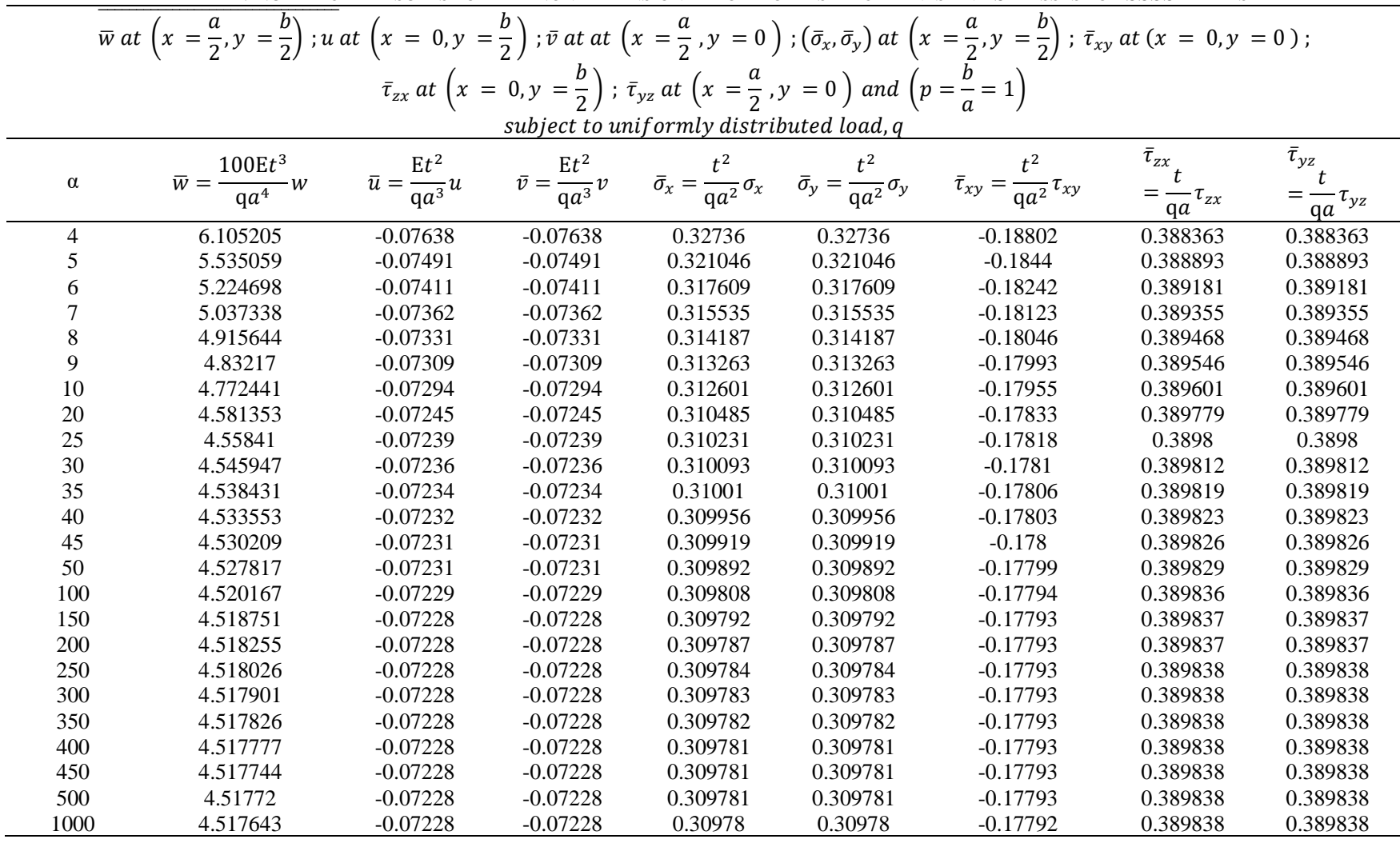


TABLE II: NUMERICAL RESULTS FOR THE NON-DIMENSIONAL FORM OF DISPLACEMENTS AND STRESSES FOR SSSS PLATES

\begin{tabular}{|c|c|c|c|c|c|c|c|c|}
\hline \multicolumn{9}{|c|}{$\begin{array}{r}\bar{w} \text { at }\left(x=\frac{a}{2}, y=\frac{b}{2}\right) ; \text { at } \\
\left(x=0, y=\frac{b}{2}\right) ; \bar{v} \text { at at }\left(x=\frac{a}{2}, y=0\right) ;\left(\bar{\sigma}_{x}, \bar{\sigma}_{y}\right) \text { at }\left(x=\frac{a}{2}, y=\frac{b}{2}\right) ; \bar{\tau}_{x y} \text { at }(x=0, y=0) ; \\
\bar{\tau}_{z x} \text { at }\left(x=0, y=\frac{b}{2}\right) ; \bar{\tau}_{y z} \text { at }\left(x=\frac{a}{2}, y=0\right) \text { and }\left(p=\frac{b}{a}=1.5\right) . \\
\text { subject to uniformly distributed load, } q\end{array}$} \\
\hline$\alpha$ & $\bar{w}=\frac{100 \mathrm{E} t^{3}}{\mathrm{~g} a^{4}} w$ & $\bar{u}=\frac{\mathrm{E} t^{2}}{\mathrm{q} a^{3}} u$ & $\bar{v}=\frac{\mathrm{E} t^{2}}{\mathrm{~g} a^{3}} v$ & $\bar{\sigma}_{x}=\frac{t^{2}}{\mathrm{q} a^{2}} \sigma_{x}$ & $\bar{\sigma}_{y}=\frac{t^{2}}{\mathrm{q} a^{2}} \sigma_{y}$ & $\bar{\tau}_{x y}=\frac{t^{2}}{\mathrm{q} a^{2}} \tau_{x y}$ & $\bar{\tau}_{z x}=\frac{t}{\mathrm{q} a} \tau_{z x}$ & $\bar{\tau}_{y z}=\frac{t}{\mathrm{q} a} \tau_{y z}$ \\
\hline 4 & 10.63138 & -0.14082 & -0.10002 & 0.530202 & 0.35911 & -0.35798 & 0.533863 & 0.355909 \\
\hline 5 & 9.92386 & -0.13997 & -0.09734 & 0.525622 & 0.352371 & -0.35198 & 0.535943 & 0.357295 \\
\hline 6 & 9.538562 & -0.13953 & -0.09585 & 0.523172 & 0.348657 & -0.34868 & 0.537107 & 0.358071 \\
\hline 7 & 9.305903 & -0.13926 & -0.09494 & 0.521709 & 0.346399 & -0.34668 & 0.537821 & 0.358547 \\
\hline 8 & 9.154761 & -0.1391 & -0.09435 & 0.520765 & 0.344925 & -0.34538 & 0.538289 & 0.35886 \\
\hline 9 & 9.051075 & -0.13898 & -0.09394 & 0.52012 & 0.343911 & -0.34448 & 0.538613 & 0.359075 \\
\hline 10 & 8.976877 & -0.1389 & -0.09364 & 0.51966 & 0.343184 & -0.34383 & 0.538846 & 0.35923 \\
\hline 20 & 8.739462 & -0.13865 & -0.09269 & 0.518197 & 0.340848 & -0.34177 & 0.539596 & 0.359731 \\
\hline 25 & 8.710953 & -0.13862 & -0.09258 & 0.518023 & 0.340567 & -0.34152 & 0.539687 & 0.359791 \\
\hline 30 & 8.695466 & -0.1386 & -0.09252 & 0.517928 & 0.340414 & -0.34139 & 0.539736 & 0.359824 \\
\hline 35 & 8.686127 & -0.13859 & -0.09248 & 0.517871 & 0.340322 & -0.34131 & 0.539766 & 0.359844 \\
\hline 40 & 8.680065 & -0.13859 & -0.09246 & 0.517834 & 0.340262 & -0.34125 & 0.539786 & 0.359857 \\
\hline 45 & 8.675909 & -0.13858 & -0.09244 & 0.517808 & 0.340221 & -0.34122 & 0.539799 & 0.359866 \\
\hline 50 & 8.672936 & -0.13858 & -0.09243 & 0.51779 & 0.340191 & -0.34119 & 0.539808 & 0.359872 \\
\hline 55 & 8.670737 & -0.13858 & -0.09242 & 0.517776 & 0.340169 & -0.34117 & 0.539815 & 0.359877 \\
\hline 100 & 8.663431 & -0.13857 & -0.09239 & 0.517732 & 0.340097 & -0.34111 & 0.539839 & 0.359892 \\
\hline 150 & 8.661671 & -0.13857 & -0.09238 & 0.517721 & 0.34008 & -0.34109 & 0.539844 & 0.359896 \\
\hline 200 & 8.661054 & -0.13857 & -0.09238 & 0.517717 & 0.340074 & -0.34109 & 0.539846 & 0.359898 \\
\hline 250 & 8.660769 & -0.13856 & -0.09238 & 0.517715 & 0.340071 & -0.34109 & 0.539847 & 0.359898 \\
\hline 300 & 8.660614 & -0.13856 & -0.09238 & 0.517715 & 0.340069 & -0.34108 & 0.539848 & 0.359898 \\
\hline 350 & 8.660521 & -0.13856 & -0.09238 & 0.517714 & 0.340069 & -0.34108 & 0.539848 & 0.359899 \\
\hline 400 & 8.66046 & -0.13856 & -0.09238 & 0.517714 & 0.340068 & -0.34108 & 0.539848 & 0.359899 \\
\hline 450 & 8.660419 & -0.13856 & -0.09238 & 0.517713 & 0.340068 & -0.34108 & 0.539848 & 0.359899 \\
\hline 500 & 8.660389 & -0.13856 & -0.09238 & 0.517713 & 0.340067 & -0.34108 & 0.539848 & 0.359899 \\
\hline 1000 & 8.660294 & -0.13856 & -0.09238 & 0.517713 & 0.340066 & -0.34108 & 0.539849 & 0.359899 \\
\hline
\end{tabular}

TABLE III: NUMERICAL RESULTS FOR THE NON-DIMENSIONAL FORM OF DISPLACEMENTS AND STRESSES FOR SSSS PLATES

\begin{tabular}{|c|c|c|c|c|c|c|c|c|}
\hline \multicolumn{9}{|c|}{$\begin{array}{r}\bar{w} \text { at }\left(x=\frac{a}{2}, y=\frac{b}{2}\right) ; \text { at }\left(x=0, y=\frac{b}{2}\right) ; \bar{v} \text { at at }\left(x=\frac{a}{2}, y=0\right) ;\left(\bar{\sigma}_{x}, \bar{\sigma}_{y}\right) \text { at }\left(x=\frac{a}{2}, y=\frac{b}{2}\right) ; \bar{\tau}_{x y} \text { at }(x=0, y=0) ; \\
\bar{\tau}_{z x} \text { at }\left(x=0, y=\frac{b}{2}\right) ; \bar{\tau}_{y z} \text { at }\left(x=\frac{a}{2}, y=0\right) \text { and }\left(p=\frac{b}{a}=2\right) . \\
\text { subject to uniformly distributed load, } q\end{array}$} \\
\hline$\alpha$ & $\bar{w}=\frac{100 \mathrm{E} t^{3}}{\mathrm{q} a^{4}} w$ & $\bar{u}=\frac{\mathrm{E} t^{2}}{\mathrm{q} a^{3}} u$ & $\bar{v}=\frac{\mathrm{E} t^{2}}{\mathrm{q} a^{3}} v$ & $\bar{\sigma}_{x}=\frac{t^{2}}{\mathrm{~g} a^{2}} \sigma_{x}$ & $\bar{\sigma}_{y}=\frac{t^{2}}{\mathrm{q} a^{2}} \sigma_{y}$ & $\bar{\tau}_{x y}=\frac{t^{2}}{\mathrm{q} a^{2}} \tau_{x y}$ & $\bar{\tau}_{z x}=\frac{t}{\mathrm{q} a} \tau_{z x}$ & $\bar{\tau}_{y z}=\frac{t}{\mathrm{q} a} \tau_{y z}$ \\
\hline 4 & 13.84533 & -0.18764 & -0.10198 & 0.669021 & 0.353675 & -0.48197 & 0.617857 & 0.308929 \\
\hline 5 & 13.02641 & -0.18666 & -0.09864 & 0.664149 & 0.347208 & -0.47255 & 0.619954 & 0.309977 \\
\hline 6 & 12.58025 & -0.18614 & -0.0968 & 0.661528 & 0.343651 & -0.46737 & 0.621117 & 0.310558 \\
\hline 7 & 12.31078 & -0.18584 & -0.09567 & 0.659957 & 0.341491 & -0.46422 & 0.621827 & 0.310913 \\
\hline 8 & 12.13569 & -0.18564 & -0.09493 & 0.658941 & 0.340082 & -0.46216 & 0.622291 & 0.311145 \\
\hline 9 & 12.01557 & -0.1855 & -0.09443 & 0.658247 & 0.339114 & -0.46075 & 0.622611 & 0.311305 \\
\hline 10 & 11.92961 & -0.18541 & -0.09406 & 0.657751 & 0.33842 & -0.45973 & 0.62284 & 0.31142 \\
\hline 20 & 11.6545 & -0.1851 & -0.09289 & 0.65617 & 0.336192 & -0.45648 & 0.623579 & 0.311789 \\
\hline 25 & 11.62147 & -0.18507 & -0.09275 & 0.65598 & 0.335923 & -0.45609 & 0.623668 & 0.311834 \\
\hline 30 & 11.60352 & -0.18505 & -0.09268 & 0.655878 & 0.335777 & -0.45588 & 0.623716 & 0.311858 \\
\hline 35 & 11.5927 & -0.18504 & -0.09263 & 0.655816 & 0.33569 & -0.45575 & 0.623746 & 0.311873 \\
\hline 40 & 11.58567 & -0.18503 & -0.0926 & 0.655776 & 0.335632 & -0.45567 & 0.623764 & 0.311882 \\
\hline 45 & 11.58085 & -0.18502 & -0.09258 & 0.655748 & 0.335593 & -0.45561 & 0.623777 & 0.311889 \\
\hline 50 & 11.57741 & -0.18502 & -0.09256 & 0.655728 & 0.335565 & -0.45557 & 0.623787 & 0.311893 \\
\hline 100 & 11.56639 & -0.18501 & -0.09252 & 0.655665 & 0.335476 & -0.45544 & 0.623817 & 0.311908 \\
\hline 150 & 11.56435 & -0.18501 & -0.09251 & 0.655654 & 0.335459 & -0.45541 & 0.623822 & 0.311911 \\
\hline 200 & 11.56364 & -0.185 & -0.09251 & 0.65565 & 0.335453 & -0.4554 & 0.623824 & 0.311912 \\
\hline 250 & 11.56331 & -0.185 & -0.0925 & 0.655648 & 0.335451 & -0.4554 & 0.623825 & 0.311912 \\
\hline 300 & 11.56313 & -0.185 & -0.0925 & 0.655647 & 0.335449 & -0.4554 & 0.623825 & 0.311913 \\
\hline 350 & 11.56302 & -0.185 & -0.0925 & 0.655646 & 0.335448 & -0.4554 & 0.623826 & 0.311913 \\
\hline 400 & 11.56295 & -0.185 & -0.0925 & 0.655646 & 0.335448 & -0.4554 & 0.623826 & 0.311913 \\
\hline 450 & 11.5629 & -0.185 & -0.0925 & 0.655645 & 0.335447 & -0.4554 & 0.623826 & 0.311913 \\
\hline 500 & 11.56287 & -0.185 & -0.0925 & 0.655645 & 0.335447 & -0.4554 & 0.623826 & 0.311913 \\
\hline 1000 & 11.56276 & -0.185 & -0.0925 & 0.655645 & 0.335446 & -0.45539 & 0.623826 & 0.311913 \\
\hline
\end{tabular}


TABLE IV: NON-DIMENSIONAL FORMS OF DISPLACEMENT AND STRESSES OF SSSS PLATE FOR $\boldsymbol{p}=\frac{\boldsymbol{b}}{\boldsymbol{a}}=\mathbf{1}$

\begin{tabular}{|c|c|c|c|c|c|c|c|c|}
\hline Study & $\alpha$ & $\bar{w}=\frac{100 \mathrm{E} t^{3}}{\mathrm{q} a^{4}} w$ & $\bar{u}=\frac{\mathrm{E} t^{2}}{\mathrm{q} a^{3}} u$ & $\bar{\sigma}_{x}=\frac{t^{2}}{\mathrm{q} a^{2}} \sigma_{x}$ & $\bar{\sigma}_{y}=\frac{t^{2}}{\mathrm{q} a^{2}} \sigma_{y}$ & $\bar{\tau}_{x y}=\frac{t^{2}}{\mathrm{q} a^{2}} \tau_{x y}$ & $\bar{\tau}_{z x}=\frac{t}{\mathrm{q} a} \tau_{z x}$ & $\begin{array}{l}\text { plate } \\
\text { theory }\end{array}$ \\
\hline Present study & & 6.105205 & -0.07638 & 0.32736 & 0.32736 & -0.18802 & 0.388363 & TRDT \\
\hline [9] & & 6.1553 & -0.0839 & 0.3597 & 0.3597 & -0.2066 & 0.3907 & TRDT \\
\hline$\%$ difference & & 0.813851 & 8.963051 & 8.990826 & 8.990826 & 8.993224 & 0.598157 & \\
\hline [10] & & 6.1044 & -0.0765 & 0.3279 & 0.3279 & -0.1883 & 0.3906 & TRDT \\
\hline$\%$ difference & & -0.01319 & 0.156863 & 0.164684 & 0.164684 & 0.148699 & 0.572709 & \\
\hline$[11]$ & & 6.1035 & -0.0769 & 0.3452 & 0.3452 & -0.1859 & 0.3849 & TSDT \\
\hline$\%$ difference & & -0.02793 & 0.676203 & 5.168019 & 5.168019 & -1.1404 & -0.89971 & \\
\hline [12] & 4 & 6.1483 & -0.0756 & 0.3392 & 0.3392 & -0.1826 & 0.3878 & TSDT \\
\hline$\%$ difference & & 0.700925 & -1.03175 & 3.490566 & 3.490566 & -2.96824 & -0.14518 & \\
\hline [13] & & 5.633 & -0.0741 & 0.287 & - & -0.1951 & 0.3331 & FSDT \\
\hline$\%$ difference & & -8.38283 & -3.07692 & -14.0627 & - & 3.628908 & -16.5905 & \\
\hline [14] & & 5.68 & -0.079 & 0.318 & - & -0.208 & 0.483 & HSDT \\
\hline$\%$ difference & & -7.486 & 3.316456 & -2.9434 & - & 9.605769 & 19.59358 & \\
\hline [15] & & 4.5201 & -0.0723 & 0.3099 & 0.3099 & -0.179 & 0 & $\mathrm{CPT}$ \\
\hline$\%$ difference & & -35.0679 & -5.64315 & -5.63408 & -5.63408 & -5.03911 & 100 & \\
\hline Present study & & 4.772441 & -0.07294 & 0.312601 & 0.312601 & -0.17955 & 0.389601 & TRDT \\
\hline [9] & & 4.7738 & -0.0741 & 0.3176 & 0.3176 & -0.1824 & 0.3818 & TRDT \\
\hline$\%$ difference & & 0.028468 & 1.565452 & 1.573992 & 1.573992 & 1.5625 & -2.04322 & \\
\hline [10] & & 4.7723 & -0.073 & 0.3127 & 0.3127 & -0.1796 & 0.392 & TRDT \\
\hline$\%$ difference & & -0.00295 & 0.082192 & 0.03166 & 0.03166 & 0.02784 & 0.61199 & \\
\hline [11] & & 4.797 & -0.0723 & 0.3243 & 0.3243 & -0.1746 & 0.3909 & TSDT \\
\hline$\%$ difference & & 0.511966 & -0.8852 & 3.607462 & 3.607462 & -2.83505 & 0.33231 & \\
\hline [12] & 10 & 4.8011 & -0.072 & 0.3233 & 0.3233 & -0.1741 & 0.3892 & TSDT \\
\hline$\%$ difference & & 0.596926 & -1.30556 & 3.30931 & 3.30931 & -3.13038 & -0.10303 & \\
\hline [13] & & 4.6701 & -0.0741 & 0.287 & - & -0.1951 & 0.3331 & FSDT \\
\hline$\%$ difference & & -2.19141 & 1.565452 & -8.92021 & - & 7.970272 & -16.9622 & \\
\hline [14] & & 4.666 & -0.075 & 0.289 & - & -0.2031 & 0.492 & HSDT \\
\hline$\%$ difference & & -2.2812 & 2.746667 & -8.16644 & - & 11.59527 & 20.8128 & \\
\hline [15] & & 4.436 & -0.0723 & 0.287 & 0.287 & -0.195 & 0 & CPT \\
\hline$\%$ difference & & -7.58433 & -0.8852 & -8.92021 & -8.92021 & 7.923077 & 100 & \\
\hline present & & 4.520167 & -0.07229 & 0.309808 & 0.309808 & -0.17794 & 0.389836 & TRDT \\
\hline$\{9\}$ & & 4.5203 & -0.0723 & 0.3099 & 0.3099 & -0.178 & 0.3802 & TRDT \\
\hline$\%$ difference & & 0.002942 & 0.013831 & 0.029687 & 0.029687 & 0.033708 & -2.53446 & \\
\hline [10] & & 4.5201 & -0.0723 & 0.3098 & 0.3098 & -0.1779 & 0.392 & TRDT \\
\hline$\%$ difference & & -0.00148 & 0.013831 & -0.00258 & -0.00258 & -0.02248 & 0.552041 & \\
\hline [11] & 100 & 4.546 & -0.0714 & 0.3203 & 0.3203 & -0.1725 & 0.3909 & TSDT \\
\hline$\%$ difference & & 0.568258 & -1.2465 & 3.275679 & 3.275679 & -3.15362 & 0.272192 & \\
\hline [12] & & 4.546 & -0.0714 & 0.3203 & 0.3203 & -0.1725 & 0.3892 & TSDT \\
\hline$\%$ difference & & 0.568258 & -1.2465 & 3.275679 & 3.275679 & -3.15362 & -0.16341 & \\
\hline$[15]$ & & 4.5201 & -0.0723 & 0.3099 & 0.3099 & -0.179 & 0 & $\mathrm{CPT}$ \\
\hline$\%$ difference & & -0.00148 & 0.013831 & 0.029687 & 0.029687 & 0.592179 & 100 & \\
\hline
\end{tabular}

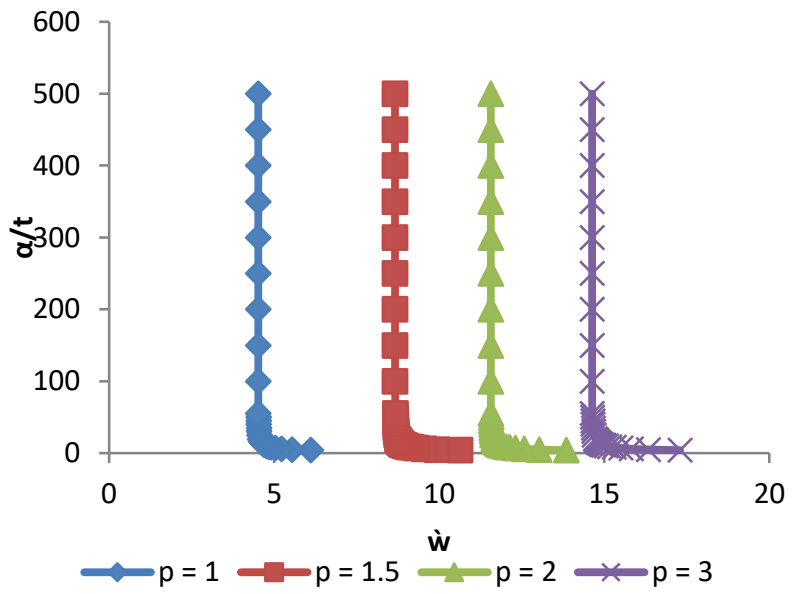

Fig 2. A graph of non-dimensional deflection against span to thickness ratio $(\alpha)$.

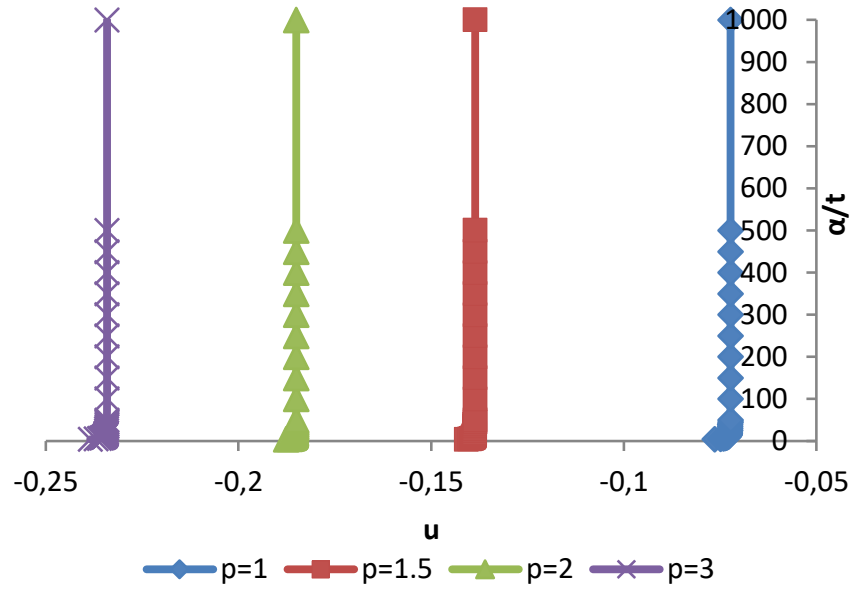

Fig 3. A graph of non-dimensional displacement against span to thickness ratio $(\alpha)$. 


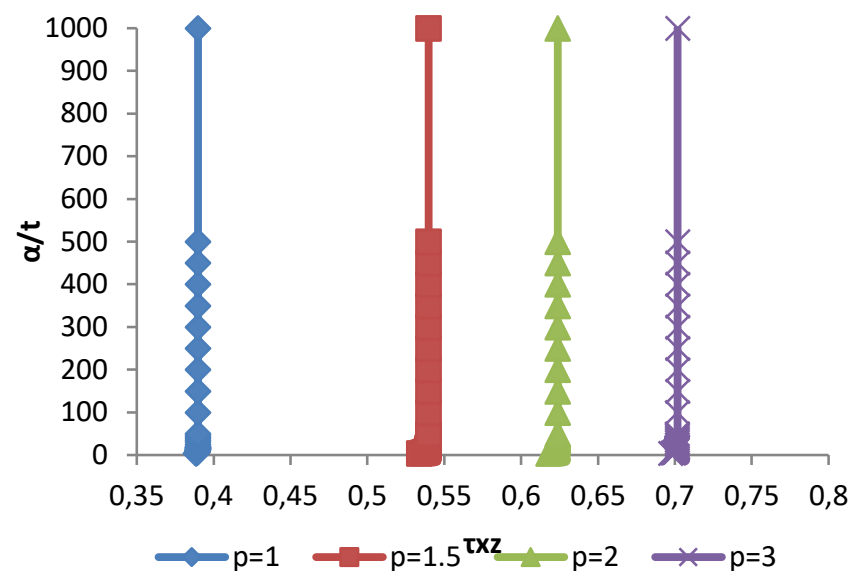

Fig 4. A graph of non-dimensional transverse shear against span to thickness ratio $(\alpha)$.

\section{ANALYSIS AND DISCUSSION}

The results obtained for the deflection at the center of SSSS isotopic thick plate, the in-plane stresses, and the vertical shear stresses at the edges of the plate for different span to depth ratio corresponding to different values of $\frac{b}{a}$ subjected to uniformly distributed load, $\mathrm{q}$ are shown in Tables $(1-4)$. It is observed that as the span-depth ratio increases, values of inplane quantities and that of out-of-plane quantities decreases for each aspect ratio, p. It can also be observed generally that as the aspect ratio increases, the values of in-plane quantities and that of out-of-plane quantities increases. However, worthy of note is that in-plane quantities being functions of $\mathrm{x}, \mathrm{y}$ and $\mathrm{z}$, vary linearly with the plate thickness but the outplane displacements, function of only $\mathrm{x}$ and $\mathrm{y}$, do not vary linearly with the plate thickness.

For the purpose of comparison in this study, results were specially generated as stated by [8]. Comparison of the results of this research and previous work done in literature is shown in Table 4 for $\propto=4,10$ and 100. it can be observed from Table 4 that the non-dimensional out of plane displacements $(\overline{\mathrm{W}})$ and in-plane displacements at $\propto=4$ as obtained in this present theory are very close to that obtained in the third order shear deformation theories (TSDT and TRDT) theories as shown but significant difference is observed in the higher order shear deformation theory (HSDT), first order shear deformation theory (FSDT) and classical plate theory(CPT) theories, however at $\propto=100$, results obtained in all the theories for the out of plane displacement and in-plane displacements can be said to be an approximation of one another. For example, the out of plane displacement $(\bar{w})$ obtained in this study at $\propto=4$ was significantly under predicted in HSDT, FSDT and CPT theories with differences ranging from $-7.486 \%,-8.38283 \%$, and $-35.0679 \%$ for HSDT [14], FSDT [13] and CPT [15] respectively. However, at $\propto=$ 100 , there is approximately no difference in the values obtained for the out of plane displacements $(\bar{w})$ and in-plane displacements in the CPT and the third order shear deformation theory used in this study. Consequently, in CPT analyses it is assumed that at span-depth ratios from 100 and above, a plate can be taken as being thin, this phenomenon is further buttressed graphically in Figures 2, 3 and 4.

\section{CONCLUSION}

Conclusions derived from this study include:

1. The theory presented in this study presents a direct, simplified, and precise approach through the use of the energy (Ritz) method resulting in Polynomial Displacement Functions to solve the problems of thick rectangular isotropic plates under transverse loading.

2. The shear deformation function derived in this study is sufficient and precise for use in the analysis of thick plates as comparison of the results of this research and previous work done in literature showed good convergence.

3. The model proposed in this study is sufficient and dependable in the analysis of isotropic thick plates since values obtained using this model showed good correlation with values obtained by other researchers using other shear deformation functions including trigonometric and polynomial shear functions and even other theories. It can also be seen that polynomial shape functions are also sufficient for analyzing thick plates.

4. The classical Kirchhoff plate theory which disregards the contributions of shear deformation under predicts the deflection at the center of SSSS thick plates with $\frac{a}{t}<100$ and hence for such cases is not a suitable theory for thick SSSS plates.

5. The deflection at the center of SSSS thick plates is significantly under predicted by HSDT [14], FSDT [13] and CPT [15] respectively. However, this percentage difference decreases as the span to thickness ratio $(\alpha)$ increases.

\section{REFERENCES}

[1] Ike CC. Kantorovich-Euler lagrange-galerkin's method for bending analysis of thin plates. Nigerian Journal of Technology, 2021; 36(2):351-360

[2] Ibearugbulem, OM, Ezeh, JC. Instability of axially compressed cccc thin rectangular plate using taylor-mclaurin's series shape function on Ritz method. Academic Research International, 2013; 4(1): 346-351.

[3] Ezeh, JC, Ibearugbulem OM, Owus M, Opara, H E, Oguaghamba OA. Galerkin's Indirect Variational Method in Elastic Stability Analysis of All Edges Clamped Thin Rectangular Flat Plates, International Journal of Research in Engineering and Technology, 2014; 3(4):674-679.

[4] Onyechere IC. Stability and vibration analysis of thick plates using orthogonal polynomial displacement functions. $\mathrm{PhD}$ Thesis. Department of Civil Engineering, Federal University of Technology, Owerri (FUTO), Nigeria. 2019.

[5] Soldatos KP. A transverse shear deformation theory for homogeneous monoclinic plates, Acta Mechanica. 1992; 94:195-200.

[6] Ventsel E, Krauthammer K. Thin Plates and Shells. New York: Marcel Decker Inc; 2001.

[7] Ugural, A. C.Stresses in Plates and Shells. 2nd Ed. Singapore: McGraw-Hills Books Co; 1999.

[8] Sayyad AS, Ghugal YM. Bending and free vibration analysis of thick isotropic plates by using exponential shear deformation theory, Applied and Computational Mechanics. 2012; 6:65-82.

[9] Onyeka, FC, Okafor, FO, Onah HN. Displacement and Stress Analysis in Shear Deformable Thick Plate. International Journal of Applied Engineering Research. 2018; 13:9893-990.

[10] Ibearugbulem OM, Ezeh J C, Ettu, L O. Energy methods in theory of rectangular plates (Use of Polynomial Shape Functions). LIU House of Excellence Ventures; 2014.

[11] Karama M, Afaq KS, Mistou S. A new theory for laminated composite plates. Proceeding of Institution of Mechanical Engineers, Series L: Design and Applications.2009; 223: 53 - 62.

[12] Krishna-Murty, AV. Towards a consistent beam theory, AIAA Journal. 1984; 22:811-816.

[13] Mindlin. R D. Influence of rotary inertia and shear on flexural motion of isotropic elastic plates. ASME Journal of Applied Mechanics. 1951; 18: $31-38$ 
[14] Reddy JN. A refined non-linear theory of plates with transverse shear deformation International Journal of Solids and Structures. 1984; 20 881-896.

[15] Kirchhoff G R. Über das Gleichgewicht and die Bewe gung einer elastschen Scheibe (in German). Journal für die reine und angewandte Mathematik.1850; 40: 51-88. 